\title{
Children with autism respond differently to spontaneous, elicited and deferred imitation
}

\author{
Mikael Heimann, Emelie Nordqvist, K. Strid, J. Connant Almrot and T. Tjus
}

\section{Linköping University Post Print}

\section{Tweet}

N.B.: When citing this work, cite the original article.

Original Publication:

Mikael Heimann, Emelie Nordqvist, K. Strid, J. Connant Almrot and T. Tjus, Children with autism respond differently to spontaneous, elicited and deferred imitation, 2016, Journal of Intellectual Disability Research, (60), 5, 491-501.

http://dx.doi.org/10.1111/jir.12272

Copyright: Wiley: 24 months

http://eu.wiley.com/WileyCDA/

Postprint available at: Linköping University Electronic Press

http://urn.kb.se/resolve?urn=urn:nbn:se:liu:diva-128738 
Running Head:

ASD and spontaneous, elicited and deferred imitation

Children with autism respond differently to spontaneous, elicited, and deferred imitation

$$
\begin{gathered}
\text { Mikael Heimann }{ }^{1} \text {, Emelie Nordqvist }{ }^{1} \text {, Karin Strid }{ }^{2} \text {, } \\
\text { Joséphine Connant Almrot }{ }^{2,3} \text {, and Tomas Tjus }{ }^{2}
\end{gathered}
$$

${ }^{1}$ The Swedish Institute for Disability Research and Division of Psychology, Department of Behavioural Sciences and Learning, Linköping University, Sweden

${ }^{2}$ Department of Psychology, University of Gothenburg, Sweden

${ }^{3}$ Psychology Unit for Maternal and Child Health, Region Västra Götaland, Sweden.

Corresponding author: Mikael Heimann, Department of Behavioural Sciences and Learning, Linköping University, SE 58183 Linköping, Sweden. Email: mikael.heimann@liu.se

Key words: Imitation, communication, autism spectrum disorder, Down syndrome 


\begin{abstract}
Background: Imitation, a key vehicle for both cognitive and social development, is often regarded as more difficult for children with autism spectrum disorders (ASD) than for children with Down syndrome (DS) or typically developing (TD) children. The current study investigates similarities and differences in observed elicited, spontaneous, and deferred imitation using both actions with objects and gestures as imitation tasks in these groups.

Methods: Imitation among 19 children with autism was compared with 20 children with DS and 23 TD children matched for mental and language age.

Results: Elicited imitation resulted in significantly lower scores for the ASD group compared with the other two groups, an effect mainly carried by a low level of gesture imitation among ASD children. We observed no differences among the groups for spontaneous imitation. However, children with ASD or DS displayed less deferred imitation than the TD group. Proneness to imitate also differed among groups: Only 10 (53\%) of the children with autism responded in the elicited imitation condition compared to all children with DS and almost all TD children (87\%).

Conclusions: These findings add to our understanding of the kind of imitation difficulties children with ASD might have. They also point to the necessity of not equating various imitation measures because these may capture different processes and be differently motivating for children with autism.
\end{abstract}


Children with autism respond differently to spontaneous, elicited, and deferred imitation

\section{Introduction}

Many studies have linked autism spectrum disorder (ASD) to problems with imitation (for current reviews, see Nadel 2014; Sevlever \& Gillis 2010; Vivanti \& Hamilton 2014). However, no consensus exists regarding the nature of this deficit or whether or not there is a general imitation deficit specific for ASD (Vanvuchelen et al. 2011). Compared to typically developing (TD) children, children with ASD often are found to imitate less frequently and less precisely and appear to have more difficulties early in development than later on (Williams et al. 2004). Some studies have found a higher probability of successful imitation for children with ASD if the tasks involve actions on objects rather than gestures (Rogers et al. 2008) or includes actions that have a clear meaning or visual goal (Gowen 2012; Rogers et al. 1996). Furthermore, imitation of simple tasks seems to be less effortful for children with ASD than imitating sequences of actions (Vivanti \& Hamilton 2014), and the "style” in which the action is presented is likely to be less imitated by children with ASD compared to TD peers (Hobson \& Lee 1999). Children with ASD are also more likely to imitate when the object is familiar and the outcome is interesting than if the object is novel or the outcome uninteresting which make it difficult to judge if the responses observed are "true imitation” in comparison with other copying processes such as mimicry and emulation (e.g., Sevlever \& Gillis 2010). Overall, the most severe problems with imitation seem to occur for imitation of non-meaningful gestures and perhaps also for body postures/kinematics (Gowen 2012; Vivanti \& Hamilton 2014).

Imitation may be spontaneous (the child imitates within an interactive flow without any direct prompts from the examiner), elicited (the examiner explicitly prompts the child: Do-as-IDo), or deferred (the child imitates a previously demonstrated action stored in long-term 
memory, not accessible by working memory). Even though children with ASD generally seem less prone to imitate than TD peers, findings indicate that they often have more difficulties with spontaneous imitation (SI) than elicited imitation (EI) (Charman et al. 2000); one must bear in mind, however, that a substantial minority of studies have failed to find any difference between children with ASD and TD children in this regard (see Vivanti \& Hamilton 2014). Various hypotheses have been put forward for why social imitation should be especially difficult for children with autism: lack of attention to social stimuli, difficulties in mapping visual representations to motor output, and a complete or partial distorted mirror system are just a few examples (e.g., Hamilton 1988; Dawson et al. 1998). From an alternative point of view, the problem with imitation overall and social imitation per se has been suggested to be secondary to the overall social difficulties central to the autism diagnosis (e.g., Heimann 1998; Nadel 2014). However, studies have used different ages and different tasks and included children with different developmental levels, making comparison across findings difficult (Colombi et al. 2009; Rogers et al. 2008). A separate reason for expecting differences between SI and EI is that they might recruit partly different brain systems, at least in adults. In a functional magnetic resonance imaging study on young adults, Guionnet and colleagues (2012) found that “instructed imitation” (read: EI) and “free imitation” (read: SI) in part activated different brain areas. More specifically, free imitation also included parts of the brain thought to be "involved in self-managing a natural interaction” (p. 974; e.g., the dorsolateral prefrontal cortex and dorsal part of the anterior cingulate gyrus).

Deferred imitation (DI) has not been investigated in ASD to the same degree as immediate imitation and the findings are diverse. Some have reported impaired DI performance compared to TD children (Dawson et al. 1998; Rogers et al. 2008; Strid et al. 2013) while some report no differences (e.g., McDonough et al. 1997). These results raise questions: Is the lower imitative 
performance often observed for children with ASD a reflection of an underlying "imitation deficit", or is the "deficit" rather connected to the recall of the modelled actions? Or is some other factor responsible for this lower imitative performance in ASD? Rogers et al. (2008) found that children with ASD had problems with both immediate and deferred imitation, suggesting that the underlying mechanisms concern imitation primarily and not memory per se.

In TD children, DI predicts both communicative and cognitive development (Heimann et al. 2006; Strid et al. 2006). For children with ASD, Toth et al. (2006) showed that DI predicted the rate of communicative development while Strid et al. $(2012,2013)$ found no differences in DI performance depending on whether the children used spoken language or not. Both groups were generally worse at DI than a matched comparison group of TD peers.

Imitation serves as an important communicative tool for the pre-linguistic child, and Nadel (2002, 2014), among others, claims that the imitative abilities of pre-linguistic children must be considered when investigating the communicative abilities in young children with ASD. According to Nadel the pragmatic ability to use turn taking, eye contact, joint attention, and imitation contributes to the TD toddler's understanding of other people's intentions, but as soon as language is mastered, the functional use of imitation as a communicative tool decreases. Thus, the problems a child with ASD might experience with an imitation task do not necessarily stem from the child's being unable to imitate. The imitative problems might, argues Nadel (2002), arise because the child does not understand the reasons why imitation of actions is expected.

The present study investigates SI, EI, and DI in a group of children with autism, some of them non-speaking. The procedure for SI and EI uses a play-like paradigm developed by Nadel (2002) while the procedure for DI builds on a paradigm outlined by Meltzoff $(1985,1995)$. Nadel's procedure consists of a series of spontaneous and elicited familiar and unfamiliar imitation tasks, gestures as well as actions with objects, with the goal to increase the likelihood 
for imitation to occur. Meltzoff's “observation-only-design” was used for measuring DI. In this procedure, the child is never allowed to handle the objects during or directly after the presentation. This prohibition is supposed to hinder the child from forming any motor representations of the action to be imitated.

The aim was to investigate if young children with ASD show less imitation compared to TD children matched on mental age and to children with Down syndrome (DS) matched on language age: We expected that (1) children with ASD generally would imitate less than both comparison groups on SI and EI tasks and less than the DS group in the DI procedure (the comparison between ASD and DI has been presented previously; Strid et al. 2013); (2) the ASD group would perform relatively better on EI tasks than on SI or DI; and (3) the non-speaking ASD subgroup would have the most difficulties with imitation. In addition, we also wanted to explore if DI performance was related to the observed SI or EI performance.

\section{Method}

\section{Participants}

Nineteen children with ASD participated (17 boys, 2 girls; Mean = 67.3 months, SD = 17.7), along with 20 children with DS (7 boys, 13 girls; Mean $=62.7$ months, SD = 12.3) and 23 TD children (11 boys, 12 girls; Mean = 35 months, SD = 5.4) (Table 1). All of the children in the ASD group were diagnosed according to the DSM-IV (American Psychiatric Association 1994) criteria by specialized neuropsychiatric teams, either at the Sahlgrenska University Hospital, Göteborg ( $n=18)$ or the Kungälv Hospital $(n=1)$. The ASD group was matched on language age with both the DS and the ASD groups and with the TD participants on mental age (Table 1). 
The neuropsychiatric work-up comprised clinically validated instruments such as the Diagnostic Interview for Social and Communication Disorders (Wing et al. 2002) or Autism Diagnostic Interview-Revised (Lord et al. 1994). For one child, diagnosed by a paediatrician, the level of autism was confirmed by administering the Childhood Autism Rating Scale (Schopler et al. 1988).

Children with ASD were divided into a speaking $(\mathrm{n}=12)$ and a nonspeaking group $(\mathrm{n}=7)$ according to the definition of phrase speech used in the Autism Diagnostic Interview-Revised: use of at least two words in combination of which one is a verb (Joseph et al. 2002). One of the authors (KS) made the categorization based on the child's use of language during the visit. A second researcher not involved in the project checked the categorization from video of five (26\%) randomly selected participants. A 100\% agreement was achieved.

Estimating mental age

Participants with ASD

For 15 of the children, information about mental age was based on the two tests used during the diagnostic work-up: Swedish versions of the Griffiths Developmental Scale (Ahlin-Åkerman \& Norberg 1991) or the Wechsler Pre-School and Primary Scale of Intelligence-Revised (Wechsler 1999). For four children, test results were either unavailable $(n=2)$ or unreliable according to the clinicians who had made the assessment $(n=2)$. For three of these children, the Raven's Progressive Matrices (Raven 1976) was used. One child never achieved a mental age estimate, and this child is not included in the calculation of the mental age for the ASD group.

\section{Participants with DS}

Thirteen children were tested with the McCarthy Scales of Children’s Abilities (McCarthy 1972) 
and seven were tested with the Griffiths Developmental Scale (Ahlin- Åkerman \& Norberg 1991; Griffiths 1970).

Participants with typical development

For the TD participants, the McCarthy Scales of Children’s Abilities was used.

\section{Estimating language age}

Because of the large heterogeneity in the language level within and among the groups, a hierarchic procedure decided which language test to use (Strid et al. 2013). The first test of choice was the Peabody Picture Vocabulary Test (PPVT-3; Dunn \& Dunn 1997); the second choice was the Swedish version of the MacArthur Communicative Development Inventories (SECDI; Berglund \& Eriksson 2000); and the final choice was the Kaufmann Expressive Vocabulary subscale (Kaufmann \& Kaufmann 1983).

Participants with ASD

Due to unwillingness to point at the pictures or not cooperating or understanding instructions, the PPVT could not be used for 11 participants, so the SECDI was used instead. For three of the participants, ceiling effects were obtained, and for these Kaufmann was used.

\section{Participants with DS}

Also here, the PPVT $(n=5)$, SECDI $(n=10)$, and Kaufmann assessment battery for children ( $n$ $=4$ ) were used in a hierarchic fashion. 
Language age was assessed by the PPVT $(n=22)$ or the Kaufmann assessment battery $(n=1)$.

Procedure

All children made two visits to the Department of Psychology, University of Gothenburg, with two or three days in between. Each visit lasted 1 to 1.5 hours, and the same researcher administered both observations for each child. The child was accompanied by at least one parent and every visit was video-monitored by two cameras at different angles covering the whole observation room.

During the two visits, several areas were studied, but here we present only results for EI and SI (e.g., Nadel 2002) and DI (Meltzoff 1985). Findings from other measures on early social and communication skills, language, and pretend play have been published elsewhere (Strid et al. 2012, 2013). This study was approved by the ethical committee at the University of Gothenburg (Ö 555-02).

\section{SI and EI}

These two types of imitation were observed during a play session that included (a) spontaneous imitation (SI) of actions or gestures performed by the examiner and (b) elicited imitation (EI) of actions or gestures initiated and prompted by the examiner (Do-as-I-do). The participants did not receive any instructions beforehand, and the length of the session depended on the child's disposition and willingness to play with the examiner. However, the mean length of this semistructured play session did not differ between the three groups of participating children $(F(2)=$ 2.748, $\mathrm{p}=.073$ ). The mean length was 15.9 minutes (range $10-29.25$ ) for the ASD group, 17.3 minutes (range 12 - 24.25) for the DS group, and 19.4 minutes (range 11.25 - 32.8) for the TD group. 
Insert Table 2 About Here

The set-up of the play session followed Nadel's (2002, 2014) instructions and included two identical sets of nine objects (Table 2). The objects were placed on a table in the room with two identical chairs next to the table. There were 11 actions (four with an object and seven predominantly non-meaningful gestures) for each of the two imitation procedures. The actions were demonstrated in a playful manner, the priority was to keep the child motivated.

The SI actions were presented without any overt instructions, and this phase always preceded the EI part. Immediately after presenting an action in the EI phase, the researcher asked the participant to repeat the action (Do-as-I-Do). If a clear imitative response was not noted after the first presentation in either the SI or EI phase, the action was repeated once.

The imitations were coded as follows (Nadel 2002): $0=$ Showed no interest in the actions or gestures performed by the examiner; 1 = Partial imitation; 2 = Correct imitation. Agreement was assessed by two researchers coding the actions independently: Cohen's kappa values were 0.93 for the SI and 0.89 for the EI.

\section{$D I$}

The DI tasks involved five actions with objects (Table 3) and the presentation followed the “observation-only-design” specified by Meltzoff (1995). Under this design the children are not allowed to handle the objects in close connection to when the action to be imitated is demonstrated, making sure that they do not develop any motor memory of the action. At the first visit, each action was presented three times during a 20-second period. At the second visit, two days later $(M=50.6 \mathrm{~h}, \mathrm{SD}=8.55)$, the participant was given the first opportunity to handle the objects in the same counterbalanced order in which they were originally presented. A 
response time of 20 seconds from when the child first touched the object was used, and the responses were later coded as $0=$ "No imitation" and $1=$ "Correct imitation" independently from videotapes by two of the authors (KS and $\mathrm{MH}$ ); the observed agreement was 0.89 (Cohen’s kappa).

Insert Table 3 About Here

Scoring and statistical analysis

The tendency to imitate was calculated from the raw imitation scores as a ratio of the maximum number of correct imitation the child would obtain divided by the total number of imitation tasks successfully presented to the child. Thus, correct imitation of all the actions would result in a value of 1 , while no imitation at all resulted in 0 .

Non-parametric tests were used due to skewness of the results for the ASD group. For overall between-group comparisons, Kruskal-Wallis was used, followed by the Mann-Whitney U test as post hoc. In addition, Friedman's two-way analysis of variance and Wilcoxon signedrank test were used for comparing between conditions. Spearman's rho was used for investigating correlations between variables. Because of the small sample sizes, two-tailed significance level was used, even though hypotheses were stated.

\section{Results}

First, a comparison of the overall imitation tendency is presented, then the group mean proportion of the different types of imitation, followed by specific group comparisons within each type. Finally, correlational analyses and imitational patterns are presented.

Overall imitation tendencies 
EI and SI were observed during a play session while DI was obtained two days later. Overall, there was a significant effect (Friedman's test, $\chi_{2}(2)=41.353, P=0.000$ ) of the type of imitation administered. Pairwise Friedman's tests (all $P<0.05$ ) revealed that the highest imitation score was obtained for DI followed by EI. The lowest level of imitation was observed for SI. Further pairwise comparisons (all $P<0.02$ ) for each group separately revealed different patterns. For the ASD group, the highest score was observed for DI, but there was no difference between SI and EI. In contrast, children with DS did not change their performance in the different imitation procedures (all comparisons ns) while the typical group displayed the same patterns as when all groups were analysed together: DI > EI > SI.

Group comparisons for each type of imitation

$S I$

We found no significant differences among groups on SI (all $P>0.05$ ). Neither did we find any significant differences in imitation scores between the speaking and non-speaking ASD subgroups $(P>0.05)$. Separate analyses comparing action with objects with gestures (Table 5) confirmed the overall results; no significant differences among the groups (all $P>0.10$ ).

Insert Tables 4 and 5 About Here

EI

At group level, there was a significant difference for EI $(H(2)=8.74, P=0.013)$ with a mean rank of 21.08 for the ASD group, 37.58 for the DS group, and 33.04 for the TD group. Pairwise comparisons using the Mann-Whitney U test post hoc revealed a significant difference between the ASD group and both the DS and the TD groups $(U=83, P=0.004$; and $U=120, P=0.03$, respectively). The DS and TD groups did not differ. Separate analyses confirmed the overall 
results for gestures only $(P=0.003)$ but not for the four tasks involving objects $(P=0.135)$.

When the ASD group was divided into speaking and non-speaking subgroups, the significant difference between groups prevailed $(\mathrm{H}(3)=12.95, P=0.005)$. Post hoc tests revealed that the non-speaking ASD group imitated less frequently than both the TD group (U = 18.5, $P=0.001)$ and the DS group $(\mathrm{U}=6, P=0.001)$. The speaking ASD group did not differ from either the TD or DS group.

\section{$D I$}

There was a significant effect of group for DI $(\mathrm{H}(2)=11.21, P=0.004)$. Post hoc analyses revealed no differences in DI between the ASD and DS groups but both groups (ASD, $P=$ 0.012; DS, $P=0.002$ ) imitated less than the TD group (note that a comparison between the TD and ASD groups has been presented previously; see Strid et al. 2013).

Splitting the ASD group into speaking and non-speaking children revealed that the observed difference between the ASD group and the TD group remained for the non- speaking group only ( $\mathrm{U}=25, P=0.004)$. No difference between the non-speaking group and the DS group was shown.

\section{Correlations}

Overall, we detected only one significant correlation: SI and EI correlated positively $\left(r_{\text {rho }}=0.475\right.$, $P=0.022$ ) for the TD group.

Willingness to imitate

To illustrate the variation among the group imitation tendencies, the pattern of not imitating is described below (see Table 6). 
Four participants (21\%) in the ASD group did not imitate any of the tasks, three of these were non-speaking. In comparison, two TD children (9\%) showed no SI at all while all children in the DS group displayed some SI.

Insert Table 6 About Here

EI

Almost half of the participants (9 out of 19) in the ASD group did not imitate in the EI condition, of whom five were in the non-speaking group. All children in the DS group did imitate while for the TD group, 3 out of the 23 participants did not imitate.

$D I$

None of the children in the ASD or TD group did not imitate DI to some degree while for the DS group only one participant failed to imitate. When the ASD group was divided into speaking and non-speaking subgroups, all of the non-speaking ASD group performed at the lower side of the scale, three or less actions. In contrast, the speaking ASD group showed a response pattern more similar to what we observed for the TD group ( $>3$ actions).

\section{Discussion}

This study explored elicited, spontaneous, and deferred imitation in children with ASD and compared their performance with children with DS and TD children. Overall, the results show different patterns for the three types of imitation studied, both when it comes to the actual imitation scores and the children's proneness to imitate. The findings partly confirm previous 
results (e.g., EI) but also provide an unexpected finding (e.g., SI).

In the EI procedure, the ASD group imitated less overall than either the TD or DS group. This finding, which is in line with much of what has previously been reported for children with autism (Vivanti \& Hamilton 2014) seemingly underscores the difficulties these children can have when explicitly asked to imitate. However, scrutiny of the findings showed that the lower EI score observed for the ASD group did not hold for the tasks involving action with objects or for the speaking subgroup. Thus, the "deficit” in EI seems primarily true for specific tasks (nonmeaningful gestures) and for children with autism who also are non-speaking.

Unexpectedly, the SI procedure yielded no significant differences between groups; the ASD group imitated to the same extent as the children with DS or the TD group. A clear majority, almost 80 percent (15 out of 19), of the children with autism did imitate to some degree. Further analyses revealed no differences among the three groups of children for imitation of gestures or actions with objects. Imitation patterns also did not differ between the speaking and non-speaking children with ASD. This finding that children with autism imitate SI to the same degree as the comparison groups is somewhat surprising because several previous studies have found that SI is especially difficult for children with ASD (see Vivanti \& Hamilton 2014 for a review). The reason for this difference between results (but see Nielsen et al. 2013 or Rogers et al. 2008 for results somewhat similar to ours) is not easy to pinpoint. It might be that the play procedure used affected the children's motivation to imitate to a lesser degree than expected. However, for this to explain the non-significant effect for SI, we have to assume that a playful imitation procedure is less motivating for TD children than for children with autism or DS. To us, this seems unlikely.

As presented elsewhere (Strid et al. 2013), the ASD group performed significantly less than the TD group on DI. New here is a comparison with children with DS, showing that children with 
ASD or DS do not differ for the types of DI tasks used in the current study. When the ASD group was divided into speaking or non-speaking subgroups, the non-speaking ASD participants differed in performance from both the TD children and the DS group. It is also noteworthy that the general tendency to imitate was higher for DI than for EI or SI. All children with ASD did display imitation, as did the TD group. Only one child belonging to the DS group failed to show any DI at all.

DI tasks might be viewed as an early declarative-like memory that later will develop into a full-blown episodic memory in Tulving's sense (Tulving \& Markowitsch 1998). However, it has been suggested that for children with ASD “the imitation demands of the test mask the child's memory ability since children with autism are described as having an 'imitation deficit'” (Strid et al. 2013, p. 30). The findings presented here complicate this view because we failed to identify a general deficit for children with autism. They performed as well as the other groups on the SI tasks, and our results point to the possibility that communicative abilities—speaking or nonspeaking —influence the observed imitation among children with autism. Finally, the DS group performed at the same overall low level as the children with ASD, which further highlights the possibility that the extent of imitation observed is more of a reflection of the child's developmental level than of the diagnosis per se.

The correlational analyses revealed no significant correlations for the ASD or DS group, indicating that the three different imitation types studied here are unrelated, at least on a behavioural level. For all groups, DI was unrelated to the other types of imitation measured, supporting the notion that DI to some extent reflects memory and not imitation.

In sum, the results indicate that language skills are related to EI and DI skills for children with ASD, in line with previous studies (e.g., Charman et al. 2000; Toth et al. 2006). The fact that the speaking ASD group did not differ from the other groups in terms of imitation indicates 
that there is no pure "imitation deficit” in ASD. Instead, the tendency to imitate varies depending on language level as well as type of task to imitate. Building on the results reported here, EI seems to be especially difficult as is also imitation of non-meaningful gestures.

A few but nonetheless important limitations must be kept in mind when evaluating the findings presented here. First, the groups were matched for mental age and language age but not for gender. There were only two girls in the ASD group compared to 13 of the children with DS and 12 of the TD group. This unevenness made it difficult to adjust for gender in our analyses. However, a comparison of gender across groups and conditions yielded non-significant results $(P$ $=0.30$ ). Second, the children were matched only on group level, and. third, the small sample size, especially for the subgroup analysis between speaking and non-speaking children with ASD, affects the generalizability of our findings.

In summary, we only partly confirmed the conclusions from recent reviews on immediate imitation that children with ASD have specific problems with imitation (Vivanti \& Hamilton 2014; Williams et al. 2004). For EI and DI, the children with ASD, especially the non-speaking subgroup, performed less imitation than the typical group. However, for SI, we found that children with ASD performed on par with the other two groups of children. Thus, the imitation difficulties often reported for children with ASD might rely on other factors such as language skills and social motivation. Especially, the non-speaking children were not helped by the social context under which imitation tasks were administered in this study.

What is missing in the literature is a more thorough clarification of whether the imitation problems often observed in ASD are the result of a lower general tendency to imitate or whether they have to do with other factors, such as less accurate imitation performance or variance in imitative response related to the specific actions that are to be imitated (e.g., Sevlever \& Gillis 2010; Vivanti et al. 2014). However, we believe that the findings presented here add 
observations that will help our understanding of how, when, and what children with autism will imitate. We also concur with Vivanti and Hamilton’s (2014) argument that non-meaningful gestures are especially difficult for children with autism, although we add that this might be true only for those who are non-speaking. 


\section{Acknowledgment/Author note}

The authors wish to thank Katarina Nilheim for generous assistance in completing this study, as well as the parents and children who participated. Preliminary versions of the results have been presented at the sixth biennial meeting of the Cognitive Development Society, San Antonio, TX, USA, October 2009, and in a Master's thesis in 2009, at the University of Gothenburg, by one of the authors (JCA).

Support for this research was provided by research grants from the Swedish Council for Working Life and Social Research, Stockholm, Sweden, to Mikael Heimann (Grant 2008- 0875), Erland Hjelmquist (Grant 2005-1700), and Tomas Tjus (Grant 2008-0518). The work of Mikael Heimann was also supported by the European Science Foundation Cooperation in Science and Technology Action (ESF COST Action) BM 1004 Enhancing the Scientific Study of Early Autism (ESSEA). The work of Emelie Nordqvist was supported by a grant from the Mayflower Research Foundation. 


\section{References}

Ahlin-Åkerman B. \& Norberg L. (1991) Griffiths utvecklingsskalor [Griffiths Developmental Scales]. Göteborg, Sweden: Psykologiförlaget [in Swedish].

Charman T., Baron-Cohen S., Swettenham J., Baird G., Cox A. \& Drew A. (2000) Testing joint attention, imitation, and play as infancy precursors to language and theory of mind. Cognitive Development 15, 481-98.

Colombi C., Liebal K., Tomasello M., Young G., Warneken F. \& Rogers S. J. (2009) Examining correlates of cooperation in autism: Imitation, joint attention, and understanding intentions. Autism: The International Journal of Research and Practice 13, 143-63.

Dawson G., Meltzoff A. N., Osterling J. \& Rinaldi J. (1998) Neuropsychological correlates of early symptoms of autism. Child Development 69, 1276-85.

Dawson G., Meltzoff A. N., Osterling J., Rinaldi J. \& Brown E. (1998) Children with autism fail to orient to naturally occurring social stimuli. Journal of Autism and Developmental Disorders 28, 479-85.

Gowen E. (2012) Imitation in autism: why action kinematics matter. Frontiers in Integrative Neuroscience 6, 117.

Griffiths R. (1970) The Abilities of Young Children. A Study in Mental Measurement. University of London Press, London.

Guionnet S., Nadel J., Bertasi E., Sperduti M., Delaveau P. \& Fossati P. (2012) Reciprocal imitation: Towards a neural basis of social interaction. Cerebral Cortex 22, 971-78.

Hamilton A. F. (2008) Emulation and mimicry for social interaction: A theoretical approach to imitation in autism. The Quarterly Journal of Experimental Psychology 61, 101-15.

Heimann M. (1998) Imitation in neonates, older infants and in children with autism: Feedback to theory. In: Intersubjective Communication and Emotion in Ontogeny: A Source Book (ed. S. 
Bråten), pp. 89-104. Cambridge University Press, Cambridge.

Heimann M., Strid K., Smith L., Tjus T., Ulvund S. E. \& Meltzoff A. N. (2006) Exploring the relation between memory, gestural communication, and the emergence of language in infancy: a longitudinal study. Infant and Child Development 15, 233-49.

Hobson R. P. \& Lee A. (1999) Imitation and identification in autism. The Journal of Child Psychology and Psychiatry and Allied Disciplines 40, 649-59.

Joseph R. M., Tager-Flusberg H. \& Lord C. (2002) Cognitive profiles and social-communicative functioning in children with autism spectrum disorder. Journal of Child Psychology and Psychiatry 43, 807-21.

Lord C., Rutter M. \& Le Couteur A. (1994) Autism Diagnostic Interview-Revised: A revised version of a diagnostic interview for caregivers of individuals with possible pervasive developmental disorders. Journal of Autism and Developmental Disorders 24, 659-85.

McDonough L., Stahmer A., Schreibman L. \& Thompson S. J. (1997) Deficits, delays, and distractions: An evaluation of symbolic play and memory in children with autism. Development and Psychopathology 9, 17-41.

Meltzoff A. (1985) Immediate and deferred imitation in fourteen- and twenty-four-month-old infants. Child Development 56, 62-72.

Meltzoff A. N. (1995) What infant memory tells us about infantile amnesia: Long-term recall and deferred imitation. Journal of Experimental Child Psychology 59, 497-515.

Nadel J. (2002) Imitation and imitation recognition: Functional use in preverbal infants and nonverbal children with autism. In: The Imitative Mind: Development, Evolution and Brain Bases (eds A. N. Meltzoff \& W. Prinz), pp. 42-62. Cambridge University Press, Cambridge.

Nadel J. (2014) How Imitation Boosts Development: In Infancy and Autism Spectrum Disorder. 
Oxford University Press, Oxford.

Raven J. C. (1976) Coloured Progressive Matrices. Oxford Psychologists Press, Oxford.

Rogers S. J., Bennetto L., McEvoy R. \& Pennington B. F. (1996) Imitation and pantomime in high-functioning adolescents with autism spectrum disorders. Child Development 67, 2060-73.

Rogers S. J., Young G. S., Cook I., Giolzetti A. \& Ozonoff S. (2008) Deferred and immediate imitation in regressive and early onset autism. Journal of Child Psychology and Psychiatry, and Allied Disciplines 49, 449-57.

Schopler E., Reichler R. J. \& Renner B. R. (1988) The childhood autism rating scale (CARS). Western Psychological Services, Los Angeles, CA.

Sevlever M. \& Gillis J. M. (2010) An examination of the state of imitation research in children with autism: Issues of definition and methodology. Research in Developmental Disabilities 31, 976-84.

Strid K., Heimann M., Gillberg C., Smith L. \& Tjus T. (2012) Deferred imitation and social communication in speaking and nonspeaking children with autism. Focus on Autism and Other Developmental Disabilities 28, 230-40.

Strid K., Heimann M. \& Tjus T. (2013) Pretend play, deferred imitation and parent-child interaction in speaking and non-speaking children with autism. Scandinavian Journal of Psychology 54, 26-32.

Strid K., Tjus T., Smith L., Meltzoff A. N. \& Heimann M. (2006) Infant recall memory and communication predicts later cognitive development. Infant Behavior and Development 29, 545-53.

Toth K., Munson J., Meltzoff A. N. \& Dawson G. (2006) Early predictors of communication development in young children with autism spectrum disorder: joint attention, imitation, 
and toy play. Journal of Autism and Developmental Disorders 36, 993-1005.

Tulving E. \& Markowitsch H.J. (1998) Episodic and declarative memory; Role of the hippocampus. Hippocampus 8, 198-204.

Vanvuchelen M., Roeyers H. \& De Weerdt W. (2011) Do imitation problems reflect a core characteristic in autism? Evidence from a literature review. Research in Autism Spectrum Disorders 5, 89-95.

Vivanti G. \& Hamilton A. (2014) Imitation in autism spectrum disorders. In: Handbook of Autism and Pervasive Developmental Disorders, Vol. 1, 4th Ed. (eds F. R. Volkmar, S. J. Rogers, P. Rhea \& K. A. Pelphery), pp. 278-301. Wiley \& Sons, Hoboken, NJ.

Vivanti G., Trembath D. \& Dissanayake C. (2014) Atypical monitoring and responsiveness to others’ goal-directed gaze in Autism Spectrum Disorder. Experimental Brain Research 232, 695-701.

Wechsler D. (1999) Wechsler Pre-School and Primary Scale of Intelligence [Swedish version] (Rev. ed.). Psykologiförlaget, Stockholm.

Williams J. H. G., Whiten A. \& Singh T. (2004) A systematic review of action imitation in autistic spectrum disorder. Journal of Autism and Developmental Disorders 34, 285-99.

Wing L., Leekam S. R., Libby S. J., Gould J. \& Larcombe M. (2002) The Diagnostic Interview for Social and Communication Disorders: background, inter-rater reliability and clinical use. Journal of Child Psychology and Psychiatry 43, 307-25. 


\section{Table 1}

Chronological age (CA), language age (LA) and mental age (MA) for the children with Autism spectrum disorder (ASD), Down syndrome (D) and the typically developing (TD) group.

\begin{tabular}{|c|c|c|c|c|c|c|c|c|c|c|}
\hline & \multicolumn{6}{|c|}{ All children } & \multicolumn{4}{|c|}{ ASD only } \\
\hline & $\begin{array}{r}A \\
(n=\end{array}$ & & & 20) & & 23) & Non- & $\begin{array}{l}\text { king } \\
\text { 7) }\end{array}$ & (n & $\begin{array}{l}\text { ing } \\
\text { 2) }\end{array}$ \\
\hline & M & $S D$ & M & $S D$ & M & $S D$ & M & $S D$ & M & $S D$ \\
\hline $\mathrm{CA}^{1}$ & 67.3 & 17.7 & 62.7 & 12.3 & 35.0 & 5.4 & 59.4 & 19.7 & 69.5 & 15.2 \\
\hline $\mathrm{LA}^{2}$ & 28.7 & 15.9 & 26.8 & 9.0 & 36.0 & 13.4 & 13.6 & $1.6^{1}$ & 36.5 & 11.8 \\
\hline $\mathrm{MA}^{3}$ & $44.5^{1}$ & 19.7 & 31.6 & 8.6 & 37.5 & 9.2 & 24.7 & 7.6 & 54.8 & 15.7 \\
\hline
\end{tabular}

${ }^{1} \mathrm{ASD}=\mathrm{DS}>\mathrm{TD}, P<0.001 ;{ }^{2} \mathrm{ASD}=\mathrm{DS}=\mathrm{TD} ;{ }^{3} \mathrm{ASD}=\mathrm{TD} ; \mathrm{ASD}>\mathrm{DS}, P=.027$. 
Table 2

Description of actions used for the spontaneous (SI) and elicited imitation (EI) conditions Imitation type $\quad$ Description $^{1}$

SI with objects

1 Umbrella: Bang on floor

4 Ball: Put on glass.

6 Glasses: Put in cup

11 Umbrella: Hold above head

SI gestures

2 Hand on ear

3 Thumb on little finger

5 Thumb on fingers with little finger stretched out

7 Thumb on mouth

8 Thumb on ring finger

9 Hand on chin

10 Open mouth + Mickey ears

EI with objects

1 Balloon: Kick with foot

4 Hat: Upside-down on head

8 Umbrella + object: Puts objects in open umbrella

11 Spoon, cup: Puts spoon in cup

EI gestures

2 Hand on head

3 V-sign with fingers

5 Thumb on middle finger

6 Thumb under nose

7 Thumb on index finger

9 Hand with palm outwards on opposite ear

10 Sticking out tongue

\footnotetext{
${ }^{1}$ Numbers represent planned order of presentation within SI and EI
} 
Table 3

Description of actions used for the deferred imitation procedure

\begin{tabular}{lll}
\hline Object & Description & Target action \\
\hline 1. Egg & Plastic egg with small beads inside & Move egg side to side 3 times \\
2. Collapsible & Cup collapses when pressed downwards & Press cup so that it collapses more than \\
cup & & hallway down \\
3. Beads & A string of beads and a plastic cup & Put string in cup \\
4. Hinge & A wooden rectangle with hinge connected & Fold the smaller wooden rectangle \\
& to it and a smaller wooden rectangle & over the larger, by using the elbow \\
& attached to the hinge & \\
5. Black box & A black box with a hidden button on top. & Press button with a pen to activate \\
& Button makes beeping sound when & sound
\end{tabular}


Table 4

Mean proportions (interquartile range) of spontaneous, elicited and deferred imitation performance for ASD, DS, and TD groups.

\begin{tabular}{|c|c|c|c|c|c|}
\hline & & ASD & & DS & \\
\hline Elicited & $0.20(0.36)$ & $0.29(0.60)$ & $0.05(0.17)$ & $0.42(0.31)$ & $0.36(0.40)$ \\
\hline Deferred & $0.57(0.25)$ & $0.64(0.40)$ & $0.46(0.40)$ & $0.52(0.20)$ & $0.74(0.20)$ \\
\hline
\end{tabular}


Table 5

Mean number of actions with objects and gestures imitated during the spontaneous (SI) and elicited imitation (EI) procedures for the three groups of participating children: autism spectrum disorder (ASD), Down syndrome (DS), and typically developing (TD).

\begin{tabular}{|c|c|c|c|c|c|c|c|c|c|}
\hline & \multirow{3}{*}{$\mathrm{N}$} & \multicolumn{4}{|c|}{ Actions with objects } & \multicolumn{4}{|c|}{ Gestures } \\
\hline & & & SI & & & & & & \\
\hline & & M & $S D$ & M & $S D$ & M & $S D$ & $\mathrm{M}$ & $S D$ \\
\hline ---speaking & 12 & 1.86 & 0.63 & 2.00 & 0.92 & 1.20 & 0.26 & 1.22 & 0.29 \\
\hline ---non-speaking & 7 & 1.50 & 0.50 & 1.07 & 0.19 & 1.1 & 0.19 & $1.0^{3}$ & 0.0 \\
\hline
\end{tabular}


Table 6

The proportion of children not imitating for the participating groups: Children with autism spectrum disorder (ASD), Down syndrome (DS) and typically developing (TD).

Mean proportion of children not imitating at all (n)

ASD DS TD

\begin{tabular}{cccccc} 
Type of imitation & All & Speaking & Non-speaking & & \\
& $\mathrm{N}=19$ & $\mathrm{~N}=12$ & $\mathrm{~N}=7$ & $\mathrm{~N}=20$ & $\mathrm{~N}=23$ \\
\hline Spontaneous & $.21(4)$ & $.08(1)$ & $.43(3)$ & 0 & $.09(2)$ \\
Elicited & $.47(9)$ & $.33(4)$ & $.71(5)$ & 0 & $.13(3)$ \\
Deferred & 0 & 0 & 0 & $.05(1)$ & 0 \\
\hline
\end{tabular}

